\title{
Chappuys e Garzoni: note sulla traduzione del “Theatro de' vari, e diversi cervelli mondani"
}

Riccardo Benedettini

\section{(2) OpenEdition}

Journals

Edizione digitale

URL: http://journals.openedition.org/studifrancesi/6509

DOI: $10.4000 /$ studifrancesi.6509

ISSN: 2421-5856

Editore

Rosenberg \& Sellier

\section{Edizione cartacea}

Data di pubblicazione: 1 septembre 2010

Paginazione: 259-274

ISSN: 0039-2944

\section{Notizia bibliografica digitale}

Riccardo Benedettini, «Chappuys e Garzoni: note sulla traduzione del "Theatro de' vari, e diversi cervelli mondani"», Studi Francesi [Online], 161 (LIV | II) | 2010, online dal 30 novembre 2015, consultato il 10 janvier 2021. URL: http://journals.openedition.org/studifrancesi/6509 ; DOI: https://doi.org/10.4000/ studifrancesi.6509

\section{(c) (i) $\odot$}

Studi Francesi è distribuita con Licenza Creative Commons Attribuzione - Non commerciale - Non opere derivate 4.0 Internazionale. 


\section{Chappuys e Garzoni: \\ note sulla traduzione del "Theatro de' vari, e diversi cervelli mondani"}

$\ll \ldots$ parecchie volte mi pare quasi avere una compagnia di persone in capo che stieno ragionando, e ogni menomo soggetto che mi si appresenti al pensiero, mi basta a farne tra me e me una gran diceria»

\section{G. LeOPARDI, Dialogo di Torquato Tasso e del suo Genio familiare (1824)}

Nel 1583 Tomaso Garzoni da Bagnacavallo (nato nel 1549, morto appena quarantenne nel 1589) pubblica a Venezia Il Theatro de' vari, e diversi cervelli mondani, edizione princeps nella quale figura il «Prologo dell'autore agli spettatori» poi sostituito, a partire dall'edizione del 1585, dal «Discorso del Cervello dell'Auttore»².

(1) IL THEATRO / DE' VARI, E DIVERSI / CERVELLI MONDANI, / NUOVAMENTE FORMATO, / ET POSTO IN LUCE / DA / THOMASO GARZONI / da Bagnacavallo. / AL CLARISSIMO SIGNORE / IL SIG. VICENZO GARZONI, / Gentilhuomo Venetiano. / CON PRIVILEGIO. / (marca editoriale) / IN VENETIA, / Appresso Paulo Zanfretti. / MDLXXXIII. Abbiamo consultato l'edizione conservata alla $\mathrm{Bi}$ blioteca Labronica «F. D. Guerrazzi» di Livorno (Villa Fabbricotti, 094 S 255). Nella numerazione, in alto a destra, delle pagine del testo $(4,118,2)$, sono presenti alcuni salti, con conseguenti omissioni nelle indicazioni dei numeri delle altre carte: si salta dalla carta 39 alla 41, senza numerare la 40 , dalla 71 alla 73 , omettendo di indicare la 72 , dalla 113 alla 116 , con omissione della 114 e 115 . Si è consultato inoltre l'esemplare a stampa del 1591 conservato nella stessa Biblioteca, IL / THEATRO / DE VARI, E DIVERSI / CERVELLI MONDANI. / Nuovamente formato, \& posto in luce / DA / THOMASO GARZONI / DA BAGNACAVALLO. / Al Clarissimo Signore / IL SIGNOR VICENZO GARZONI, / Gentil'huomo Venetiano. / CON PRIVILEGIO. / (marca editoriale) / IN VENETIA / Appresso Gio. Battista Somasco. 1591, (Villa Fabbricotti, 094 S 122), rilegato all'interno di un volume che contiene l'edizione veneziana del 1586 dell'Hospidale de' pazzi incurabili. Anche nella numerazione, sempre in alto a destra, delle carte $(4$, 96, 2) di questa ed. 1591 compaiono alcuni errori: si ripetono i numeri 35-36, e si ha una successione errata a cominciare dalla carta $67: 67,68,67,70$, 69, 70, 71 .

(2) Salvo diverse indicazioni, nostro testo di riferimento per il raffronto linguistico con la traduzione francese, è Il teatro dei vari e diversi cervelli mondani presente nelle Opere di Tomaso Garzoni a cura di P. Cherchi (Ravenna, Longo Editore, 1993), dove si adotta come testo base l'edizione veneziana del 1585 per i tipi di G. B. Somasco. Abbiamo consultato inoltre i tre esemplari a stampa conservati presso la Biblioteca $\mathrm{Na}$ zionale Centrale di Firenze: IL THEATRO / DE' VARI, E DIVERSI / CERVELLI MONDANI, / Nuovamente formato, \& posto in luce / DA / THOMASO GARZONI / DA BAGNAVALLO. / $\mathrm{Al} \mathrm{Cl} .{ }^{\mathrm{mo}}$ Signore / Il Signor Vicenzo Garzoni, / Gentil'huomo Venetiano. / CON PRIVILEGIO. / (marca editoriale) / In Venetia, Appresso Fabio, \& Agostin Zoppini, fratelli. 1585. (Magliabechiano 3.1.230), copia nella quale non compaiono errori di numerazione $(118,5)$; IL THEATRO / DE' VARI, E DIVERSI / CERVELLI MONDANI. / NUOVAMENTE FORMATO, ET / posto in luce da TOMASO Garzoni / da Bagnacavallo. / AL CLARISS. SIG. IL SIGNOR / VINCENZO GARZONI, / Gentil'huomo Venetiano. / Aggiuntovi di nuovo il cervello dell'Autore. / (marca editoriale) / IN FERRARA, / Appresso Giulio Cesare Cagnacini, \& Fratelli. / Con licenza de' Superiori, 1586. (Magliabechiano V.10.223): in questa copia, dove non si hanno errori nella numerazione delle pagine $(8,151)$, la carta 145 è stata tagliata; IL / 
Garzoni era un canonico regolare lateranense che, nella sua attività di predicatore itinerante, seppe ben individuare le tematiche di interesse e di studio per il pubblico dei lettori; inesauribile poligrafo, spostandosi tra Bagnacavallo, Ravenna e la Ferrara degli Estensi, ma con contatti anche con Mantova, Treviso e Venezia, egli era convinto che scrivere significasse innanzitutto divulgare. La divulgazione appariva in forma compiuta quanto più articolato, sviluppato e classificato risultava il sapere con il quale si presentava il materiale, secondo un atteggiamento culturale coerente con la sensibilità, il gusto, la coscienza storica dell'epoca alla quale il romagnolo apparteneva'.

Prodotta in soli sei anni, la fittissima opera di questa «figura singolarissima, eminentemente teatrale, creatore inesauribile di 'scene immaginarie' e di 'teatri di meraviglie' (si definiva 'giullare')», ebbe una grande risonanza fino al Seicento inoltrato, forse proprio per quel linguaggio, già seicentesco, capace di portare «all'aria aperta, quasi sulla scena, nelle sue espressioni popolari e borghesi, la società del tempo», come incisivamente ha scritto Giovanni Macchia. Riprova dell'interesse e della modernità delle sue opere è dato, oltre che dalle numerose edizioni italiane pubblicate in pochi anni, dalle varie traduzioni in francese, in inglese, in spagnolo e in tedesco, trasparente esempio di una ricezione che tende a varcare i confini.

La traduzione francese del Theatro de' vari, e diversi cervelli mondani ad opera di Gabriel Chappuys (1546-1612 o '13) ${ }^{4}$, uscita a Parigi nel 1586 per i tipi di Felix Le Mangnier con privilegio reale di nove anni concesso al libraio Jean Houzé e datato

THEATRO / DE VARI, E DIVERSI / CERVELLI MONDANI. / Nuovamente formato, \& posto in luce / DA / THOMASO GARZONI / DA BAGNACAVALLO. / Al Clari imo Signore / IL SIGNOR VICENZO GARZONI. / Gentil'huomo Venetiano. / CON PRIVILEGIO. / (marca) / IN VENETIA, / Appresso Gio. Battista Somasco. 1591. (Magliabechiano V.5.233), esemplare nel quale si hanno varî errori di numerazione $(4,96$, 2): dall'errata indicazione della carta 35 e sg. (35, $36,35,36,37,38)$ deriva un errato conteggio delle carte dei Discorsi interessati: XIX $(34 \mathrm{v}-35 \mathrm{r}), \mathrm{XX}$ (35v), XXI (36r), XXII (36v-35r), XXIII (35r-35v), XXIIII (35v-36r), XXV (36r-36v); come già segnalato per l'esemplare conservato a Villa Fabbricotti, anche in questa copia si ripetono gli errori presenti nella serie delle carte 67, 68, 67, 70, 69, 70, 71 .

(3) Sulla biografia di Garzoni sappiamo poco. Si può vedere il Laconismo vitale circa l'autore premesso dal fratello Bartolomeo all'opera di Tomaso Il Serraglio de gli Stupori del Mondo, testo edito postumo solo nel 1613, e oggi in appendice a T. GARZONI, Le vite delle donne illustri della scrittura sacra. Con l'aggionta delle vite delle donne oscure e laide dell'uno e l'altro Testamento. E un Discorso in fine sopra la nobiltà delle donne, a cura di B. CollinA, Ravenna, Longo Editore, 1994, pp. 249251, volume cui si rinvia anche per l'introduzione. Si veda inoltre Dizionario Biografico degli Italiani, Istituto dell'Enciclopedia Italiana, Roma, Treccani, 1960-, vol. 52, 1999, ad vocem a cura di O. NiCCOLI, pp. 449-453. Sul carattere enciclopedico o repertoriale delle opere di Garzoni, si veda P. CHERCHI, Enciclopedismo e politica della riscrittura: Tommaso Garzoni, Pisa, Pacini, 1980 e ID., Polimatia di riuso. Mezzo secolo di plagio (1539-1589), Roma, Bulzoni Editore, 1998.
(4) Per notizie su Gabriel Chappuys, si rimanda a: L. Bolzoni, Il mondo utopico e il mondo dei cornuti. Plagio e paradosso nelle traduzioni di Gabriel Chappuys, «I Tatti Studies. Essays in the Renaissance», volume 8, 1999, Florence, Olschki, pp. 171196; J. Balsamo, Autour de Gabriel Chappuys: quelques éléments pour une typologie des traducteurs au $X V I^{e}$ siècle, in «Franco Italica», 10, «Auteurs traducteurs entre France et Italie», 1996, pp. 1-10; A. PREDA, L'opera bizzarra di Tommaso Garzoni e la traduzione di Gabriel Chappuys: un'ambigua demistificazione della pazzia, in «Studi di Letteratura francese», XIX, «Cinquecento visionario tra Italia e Francia», Firenze, Olschki, 1992, pp. 185-197; P. DE CAPITANi, Da 'pedante' a 'poeta': la figura dell'uomo di lettere nei "Dialoghi piacevoli" di Nicolo Franco tradotti da Gabriel Chappuys, ivi, pp. 199-214, segnatamente nota 30 p. 209; EAD., Un traducteur français de textes italiens à la fin de la Renaissance. Gabriel Chappuys (env. 1546-env. 1613), in «Filigrana», n. 6, 2000-2001, I, «La Lettre, le Secrétaire, le Lettré. De Venise à la cour d'Henri III. Francesco Sansovino - Gabriel Chappuys», HURBI, Département d'études italiennes - Université Stendhal-Grenoble 3, pp. 89-114. Utili indicazioni si ricavano oggi nei varî contributi raccolti in «Il segretario è come un angelo». Trattati, raccolte epistolari, vite paradigmatiche, ovvero come essere un buon segretario nel Rinascimento, Atti del XIV Convegno Internazionale di Studio, Verona, 25-27 maggio 2006, a cura di R. GORRIS CAMOS, con la collaborazione di S. Arena e L. Colombo, Fasano, Schena Editore, 2008, segnatamente V. Mellinghoff-Bourgerie, Le "Secrettaire" de Gabriel Chappuys, face au "Del Secretario" de Francesco Sansovino et à "The English Secretary" d'Angel Day. Remarques sur l'béritage de l'éthos épistolographique érasmien, pp. 63-91. 
7 febbraio $1586^{5}$, è parte significativa della diffusione del testo italiano. Traduttore dal latino, italiano e spagnolo, lavorando prima per editori di Lione poi per altri di Parigi, anche Chappuys è mosso da una volontà di divulgazione, da un desiderio di essere letto che lo porta ad operare su testi di ampia fortuna: si ricordi soltanto la traduzione di alcuni libri dell'Amadis de Gaula, quella dell'Orlando furioso, del Cortigiano, della Civile Conversazione e della Fiammetta. ${ }^{6}$ In quest'ottica, l'interesse per il catalogo dei «cervelli» («intendendo per il cervello l'ingegno», Discorso proemiale, 49) del Garzoni era stato preceduto, nel 1580, da quello per la traduzione di un'opera spagnola quasi coeva e di analogo argomento contemporaneo: l'Examen de Ingenios para las ciencias (I ed. Baeza, 1575) ${ }^{7}$, dell'ebreo converso Juan Huarte, filosofo naturale che esercitò la professione di medico in Navarra e in Catalogna. Non possiamo documentare che Garzoni avesse consultato il testo del medico spagnolo, già tradotto in italiano nel 1580 da Camillo Camilli a Venezia, per i tipi di Aldo Manuzio, con il titolo Essame de gl'ingegni de gli buomini, per apprender le scienze. La titolatura delle due opere dimostra una trasparente affinità, ma diverse appaiono le esigenze che ne sottendono il contenuto, dalle prospettive letterario-divulgative per il Theatro a quelle più psico-fisiologiche per l'Examen ${ }^{8}$. Con la sua attenzione a ricondurre la follia in una dimensione culturale scientifica, Huarte sembra in contrasto con l'analisi filosofica e morale che Garzoni dà dell'«incurabile malattia». Probabilmente, tale contrasto è aggirabile tenuto conto che l'orientamento di fondo di entrambe le

(5) LE / THEATRE DES / DIVERS CERVEAUX / DU MONDE. / AUQUEL TIENNENT PLACE, / selon leur degré, toutes les manieres d'esprits et / humeurs des hommes, tant louables que vicieuses, / deduites par discours doctes et agreables. / Traduict d'Italien, par G.C.D.T. / (marca editoriale) / A PARIS, / Pour Felix le Mangnier, Libraire Iuré en l'Uni- / versité de Paris, au Palais en la Gallerie al- / lant à la Chancellerie. / M. D. LXXXVI. / AVEC PRIVILEGE DU ROY. $\mathrm{Si}$ è consultato l'edizione presente sul sito on-line www.bvh.univ-tours.fr elaborata dal CESR con la collaborazione dell'IRHT. Il testo, costituito di 268 carte in-16, presenta vari errori di numerazione: la «Table des Discours» e quella «des Auteurs» (rispettivamente di $6 \mathrm{cc}$. ciascuna) mancano di numerazione; si ha una serie errata a partire dalla $\mathrm{c}$. 106r: 106r, 106v, 109r, 109v, 108r, 108v, 111r, 111v, 110r, 110v, 113r, 113v, 112r, 112v, 113r, 113v, 114r; si salta da $144 \mathrm{v}$ a $149 \mathrm{r}$, procedendo con la 150 (non si numerano dunque le cc. 145-148); si ripete due volte la numerazione $164 \mathrm{r}$ e $164 \mathrm{v}$. Per quanto riguarda la numerazione dei Discours, si numera 36 il Discours 35 [«DISCORSO XXXV / De' cervelloni saggi e gravi» $(156,67 r) \rightarrow$ «DES GRANDS CER- / veaux et entendemens sages et / graves. / DISCOURS XXXVI» (154r)], quindi il 36 diventa 37 [«DISCORSO XXXVI / De' cervelloni cabalistici» $(160,69 \mathrm{r}) \rightarrow$ «DES CERVEAUX ET / grands entendemens Caba- / listiques.» (160r)], mentre col 37 si ritorna alla corretta numerazione: «DISCORSO XXXVII / De' cervellazzi rozzi ed incivili» $(164,71 \mathrm{v}) \rightarrow$ «DE CEUX QUI SONT / du tout sans cervelle, lour- / dauts et incivils.» (164r).

(6) Per un'accurata bibliografia dei lavori di Chappuys, vedi: P. DE CAPITANI, Bibliographie des ouvres de Gabriel Chappuys, in «Filigrana», op. cit., pp. 187-194; P. Mula, Note bibliographique sur Gabriel Chappuys, ivi, pp. 195-198. Sull'esprit delle traduzioni francesi di testi italiani, si veda J. Balsamo, Les rencontres des Muses. Italianisme et anti-italianisme dans les Lettres françaises de la fin du XVI siècle, Genève, Slatkine, 1992 e ID., Traduire de l'italien. Ambitions sociales et contraintes éditoriales à la fin du XVIT siècle, in Traduire et adapter à la Renaissance, Actes de la journée de l'École des Chartes, éd. par D. De Courcelles, Paris, Études et rencontres de l'École des Chartes, 2, 1998, pp. 89-98.

(7) J. Huarte de San Juan, Examen de ingenios para las ciencias, Introducción, edición y notas de E. Torre, Barcelona, PPU, 1988; si veda anche l'edizione italiana Esame degli ingegni, a cura di R. Riccio, Bologna, Editrice CLUEB, 1993. Il testo, diviso in 18 capitoli preceduti da un Proemio dell'Autore e un Proemio per il lettore, è messo all'Indice nel 1583, tradotto in italiano nel 1582 (i numerosi esemplari presenti ad oggi in varie biblioteche italiane ne provano l'ampia fortuna) e in inglese nel 1594; la sua ristampa in Spagna nel 1594, con l'aggiunta di nuovi capitoli, serve per le ristampe di Alcalá de Henares, di Anversa e per le varie riproposte spagnole ed olandesi impresse nel Seicento. La traduzione di Chappuys, edita a Lione nel 1580 per i tipi di Etienne Brignol, si intitola Anacrise, ou parfait jugement et examen des esprits propres et naiz aux sciences. Per le date delle numerose riedizioni, si veda la «Bibliografia» redatta da E. TORRE, op. cit., p. 51 .

(8) Si veda la premessa di R. RICCIO alla sua traduzione in italiano moderno dell'Examen, op. cit., p. 27 e sg. E di «discorso morale anziché fisiologico o scientifico» scrive anche P. CHERCHI nella sua «Introduzione» al testo, op. cit., p. 42. 
opere si basa sulla concezione umorale della follia; comunque Garzoni scriveva da «frate spregiudicato» (Bronzini); di lì a poco la sua città contemporanea, infestata dalle mille professioni e mestieri (in realtà cinquecentoquaranta), sarà ostracizzata dal mantovano Antonio Possevino, della Compagnia di Gesù', il quale riconosce i dissensi religiosi che questo nuovo «giullare della scienza» si affretta a celare sotto il manto della Chiesa ${ }^{10}$. Il problema che si potrebbe porre è in breve quello relativo alla mutata concezione della follia; ma, per affrontare la questione, bisognerebbe prima soffermarsi sull'esperienza di inizio Cinquecento, che vede la follia diventare essa stessa una forma di saggezza, quindi, in pieno post-tridentino, il suo calarsi in una dimensione patologica: sarà opportuno lasciarla da parte. Possiamo invece affermare che entrambi questi tentativi classificatori ${ }^{11}$, costruiti sull'uso di documentate e ordinate citazioni mediche, quanto sull'accumulo di insalate di parole dal vago sapore schizofrenico («Et inveniunt similes labra lactucas», scrive Erasmo), contribuiscono così a dare un volto a quella follia che troverà stabile riscontro nei racconti di Cervantes e nei drammi di Shakespeare.

Per la traduzione del Theatro di Garzoni, Chappuys utilizzò un esemplare del $1583^{12}$, come si vede non solo dal fatto che egli rende il Prologo in «PREFACE, / LE THEATRE DE / l'Hauteur aux spectateurs.» (12 carte con varî errori di numerazione: 8r-v, 4r-v, 5r-v, 6r-v, 7r-v mancano di indicazione numerica, 8r-v $)^{13}$, ma anche dall'assenza di un errore nella traduzione, scarto che si sarebbe potuto verificare solo se il traduttore si fosse basato sul Discorso proemiale dell'ed. 1585, dove compare la forma temuto anziché tenuto. Garzoni, secondo un'impronta di stampo erasmiano (l'amor di se stessi, caratteristica specifica di tutti, è considerato il primo indizio della follia e, non per caso, Filautía è la prima delle compagne che Follia trascina con sé

(9) Anche il Possevino propone una classificazione dei «cervelli», o «ingegni» che dir si voglia. Nel 1598 a Vicenza, presso Giorgio Greco, il gesuita pubblica, in traduzione italiana, la Coltura de gl'ingegni - recensione o discussione dell'Examen di Huarte -, edizione autonoma di una parte della sua opera Bibliotheca Selecta (I ed., Roma, Tipografia Apostolica Vaticana, 1593, 2 voll.). Vedi A. Possevino, Coltura de gl'ingegni (Vicenza, 1598), Ristampa anastatica, Postfazione di A. ArCANGELI, Bologna, Arnaldo Forni Editore, 1990; A. BIONDI, La Bibliotheca Selecta di Antonio Possevino. Un progetto di egemonia culturale, in La «Ratio Studiorum». Modelli culturali e pratiche educative dei Gesuiti in Italia tra Cinque e Seicento, a cura di G. P. BrizzI, Roma, Bulzoni, 1981, pp. 43-75; L. Balsamo, Venezia e l'attività editoriale di Antonio Possevino (1553-1606), in «La Bibliofilía. Rivista di storia del libro e di bibliografia», Anno XCIII, 1991, Disp. 1, pp. 53-93.

(10) T. GARZONI, La piazza universale di tutte le professioni del mondo, a cura di G. B. BRONZINI, con la collaborazione di P. DE MEo e L. CARCERERI, Firenze, Leo S. Olschki Editore, 1996, Biblioteca di «Lares», Nuova Serie, vol. XLIX, Monografie, vol. I-II, p. IX e sg. dell'Introduzione (pp. VIIXLIV).

(11) «La folie n'est plus qualifiée, par son déplacement, en métaphore, mais quantifiée, par son démembrement analytique, en diminutifs et péjoratifs: elle n'est plus une absence d'oubli, mais une encombrante présence à mesurer», osserva al riguardo C. Ossola, Métaphore et inventaire de la folie dans la littérature italienne du XVI siècle, in Folie et déraison à la Renaissance, Colloque international tenu en novembre 1973 sous les auspices de la Fédération Internationale des Instituts et Sociétés pour l'Étude de la Renaissance, «Travaux de l'Institut pour l'étude de la Renaissance et de l'Humanisme V», Bruxelles, Éditions de l'Université de Bruxelles, pp. 171-196: p. 190.

(12) Consentono qui i curatori del Théâtre online e M. TETEL, La morosophie transalpine de Garzoni: diversion, subversion, non-traduction, in «Studi di Letteratura francese», XIX, op. cit., pp. 215 227: p. 218. Dissente P. CHERCHI, secondo il quale Chappuys si sarebbe basato sull'edizione riveduta e ampliata del 1585 (op. cit., p. 45). Che Chappuys abbia lavorato sull'ed. italiana del 1583 o su quella del 1585 - o che abbia potuto consultare entrambe -, risulta comunque poco pertinente ai fini della nostra analisi, se si esclude ovviamente la rilevanza del Discorso liminare presente nella traduzione francese.

(13) Il «PROLOGO / IL THEATRO / DELL'AUTTORE / A'SPETTATORI» (ed. 1583, cc. $1 \mathrm{r}-4 \mathrm{r}$ ) contiene la dichiarazione di intenti dell'«Architetto» (4r) Garzoni, il quale, con questo «Theatro Reggio, \& Signorile» (4r) scrive di aver «voluto nondimeno con altissimo ardimento, tentar di fabricare un Theatro, non però materiale, ma intellettuale per molte conditioni (rimettendosi al giudicio de gli altri) o pari, ò superiore à quelli degli antichi» (1v). 
nella danza), inizia l'opera parlando di coloro i quali sono convinti della propria grandezza o importanza: «E questa loro sciagura non procede da altro più propriamente che dal tenersi troppo da se stessi, perché non bisogna tenersi, ma esser tenuti, overo con gli effetti mostrare al mondo che l'uomo almeno debba esser tenuto» (47; nel testo base si ha temuto ma è Cherchi, come leggiamo nella nota critica, a correggere, giustamente, la forma in tenuto). Nell'ed. 1583 (e poi anche in quelle del 1591 da noi consultate) l'errore non compare, «che l'huomo almeno debba esser tenuto» (5v). E ben traduce Chappuys, «et ceste leur infortune ne procede plus proprement d'autre chose, que de se reputer trop eux mesmes: car il ne faut pas se reputer, mais estre reputez, ou bien monstrer par effects au monde, que / l'homme, au moins doive estre tenu et reputé» $(17 \mathrm{v}-18 \mathrm{r})$. Secondo una modalità di duplicazione che vedremo essere frequente nella traduzione, Chappuys fa precedere il participio reputé proprio da tenu, forma che conserva il calco italiano in un francese senz'altro più italianizét ${ }^{14}$.

Nell'epistola dedicatoria «A NOBLE ET TRES VER / tueux, PIERRE HABERT / Conseiller du Roy, Secretaire de la Chambre, de ses Finances, \& de la / Maison \& Coronne de France: Bailly / de son Artillerie, \& Garde du scel d'icelle \&c.» (2r-2v, cc. non numerate), datata 20 maggio 1586, Gabriel Chappuys motiva la propria traduzione con ragioni di «plaisir et contentement» per il lettore ${ }^{15}$ : il carattere piramidale del Teatro, nel quale ogni cervello occupa un seggio ben preciso, permetterà al pubblico di dilettarsi nell' «assigner lieu propre et peculier, à un nombre infiny d'autres cerveaux, de quelque humeur qu'ils soient menez». Lo spettacolo offerto dal Theatro «nobilissimo» (Prologo, ed. 1583, 3r) è ben moderno e, per ragioni che è facile intuire, adatto anche alla «Court du plus grand, magnanime et Religieux Prince du monde, entre autres, ce Phoenix des bons Cerveaux». E quando, nelle parole conclusive della dedica, Chappuys scrive «Ie prie Dieu de changer tellement les bijarres cerveaux des hommes, qu'en fin l'heresie face place à la religion, le vice à la vertu, l'hypocrisie à la verité, la simulation à la sincérité, et les mauvais aux bons», si ha l'impressione che la Follia sia paradossalmente fuggita da uno dei suoi numerosi, «amplissimi» (ed. 1583, 2r) e, per quanto possibile, organizzati seggi.

La scrittura di Garzoni risente di quegli elementi stilistici che col tempo si sono sempre più radicati nel gusto letterario dell'epoca: gli aneddoti a grappoli, il ricorso alle citazioni della Bibbia, a quelle dei Padri e dei Dottori della Chiesa, degli autori latini e greci, dei classici italiani. E anche quando la citazione non è riportata per esteso, lo scrittore non trascura il nome della fonte cui attinge, il tutto per risplendere in quel registro erudito che si associa a temi storico-filologico-letterarî: è allora convenzione diffusa che «l'occultamento non è il cuore del plagio» ${ }^{16}$. La fedele traduzione di Chappuys, pur non chiudendosi di fronte a questo orizzonte, vi si apre solo in parte; ma questo non significa che egli manchi nel proprio compito di traduttore-divulgatore ${ }^{17}$. Chappuys probabilmente si diverte nel cercare il corrispettivo di ogni parola usata nei lunghi cataloghi di Garzoni: poche, lo vedremo, sono le esclusioni dei termini tecnici o specialistici italiani e, quando tali scarti si verificano, essi riguardano delle parole utilizzate per menzionare le complesse rassegne di armi, nonché alcune cognizioni

(14) Qualche dato sul concetto della «sororità» del francese e dell'italiano si ha in P. CHERCHI, Corbinelli, Postel e il problema dell'antico toscano, in Guillaume Postel 1581-1981, Actes du Colloque International d'Avranches 5-9 septembre 1981, Paris, Guy Trédaniel, éd. de La Maisnie, 1985, p. 320 e sg.

(15) Sul «plaisir» che motiva Chappuys anche nella traduzione dei Mondi del Doni, rimandiamo a L. BOLZONI, art. cit., p. 180.

(16) Vedi, oltre a P. CHERCHI, Polimatia di riuso, op. cit., R. A. POSNER, Il piccolo libro del plagio, Roma, Elliot Edizioni, 2007, p. 23 (il non lo aggiungiamo noi). Sempre a POSNER si rinvia per i concetti di «imitazione creativa» (p. 57) e di non importanza dell'«originalità» (p. 67).

(17) Vedi A. PrEDA, art. cit., pp. 196-197. 
proprie dell'alchimia. Per quanto riguarda le numerose citazioni (siano esse tratte da fonti latine, greche o italiane), la cui funzione di «riscrittura» e di dar «voce» alla follia è chiara in Garzoni, Chappuys decide di riportare il passo per intero, talvolta traducendolo in francese, come di ridurlo, persino di ometterlo nella sua interezza, senza che si possa trovare, crediamo, un vero e proprio criterio univoco di scelta, se non un desiderio di sintesi in grado di rendere il testo meglio godibile ad un ampio, e non necessariamente colto, pubblico francese. In questo modo ci pare possano interpretarsi anche alcune semplificazioni di patronimici dalla valenza classica, ogni volta sciolti con cura dal traduttore. Metteremo dunque in luce qualche costante della traduzione, nella scelta del vocabolario e delle immagini, procedendo da un elemento di novità che è proprio del testo di Chappuys: la grande varietà di espressioni utilizzata per rendere in francese il sostantivo «cervello» e le sue varianti.

\section{Classificazione dei cervelli}

Chappuys procede ad una vera e propria analisi dei cervelli, che qui si cercherà di riassumere. Nel riportare la «TABLE DES DIS / COURS.», il traduttore rende ogni volta con «Des cerveaux» i tanti «De' Cervelli, De' Cervellini, De' Cervelluzzi, De' Cervelletti, De' Cervelloni, De' Cervellazzi ${ }^{18}$. Si considerino solo due esempî: «Discorso II. De'cervelli bravi ed armigeri» $(60) \rightarrow$ «Des cerveaux braves, valeureux et belliqueux. Discours II.» (27v); «Discorso XI. De' cervellini vani» $(87) \rightarrow$ «Des cerveaux vains. Discours XI» (61r). Per quel che riguarda invece la titolatura dei sei gruppi, il primo, quello dei «CERVELLI» (Discours 1-10), manca di titolo; gli altri cinque gruppi, per i quali Chappuys conserva lo schema originario, hanno come titoli: «Legers de Cerveau.» (Disc. 11-15), «Ecervellez.» (Disc. 16-24), «Cerveaux petits.» (Disc. 25-28), «Cerveaux grands.» (Disc. 29-36), «Sans cervelle du tout.» (Disc. 37-55). Se per i Discorsi relativi ai «Cervelli», la traduzione, lo si è detto, è sempre «Cerveaux» - sia che il termine compaia nei titoli dei capitoli, sia nel contenuto del capitolo stesso -, significative variazioni si hanno invece per gli altri tipi di cervelli. Tentiamo di procedere ad una classificazione sulla base della presenza del termine nel titolo o nel contenuto del Discours stesso.

I «cervellini» sono così evocati: «Altri, diminuendo alquanto dalla sua perfezione, diminuiscono ancora del vocabolo e meritano il nome più presto di cervellini, onde nell'idioma latino si ritrova il vocabolo cerebrosus che significa cervellino ovvero di cervello leggiero» (49-50) $\rightarrow$ «Les autres diminuans un peu de ceste perfection, diminuent aussi du vocable, et s'appellent Cerveaux legers, en Italien, Cervellini, d'où, en la langue Latine, se trouve le terme, de Cerebrosus» (21r). Chappuys traduce la parola in francese, conservando la spiegazione della genesi del termine. E al più generico «Cerveaux» egli ricorre ancora nei titoli dei Discorsi dove Garzoni scrive dei «Cervellini» (XI, XII, XIII, XIV - l'omissione della resa in francese di «spuzzetti» non si avrà invece nel corso del capitolo, laddove l'aggettivo qualificante il sostantivo è tradotto in «delicats»-, XV). Nel Discours la traduzione di «cervellini», resa quasi sempre in «cerveaux», può conservare, esplicitandola, la forma italiana («chiamati cervellini», $87 \rightarrow$ «appellez en Italien, Cervellini, comme eventrez ou legers de cer-

(18) Contrariamente all'ed. francese, che riporta la Table nelle prime pagine del testo, entrambe le edizioni italiane $(1583,1585)$ inseriscono la «TAVOLA DELLI DISCORSI.» nelle ultime pagine (119r-v, mancanti di indicazione numerica). Segue la «TAVOLA DE GLI SCRITTORI / ALLEGATI NELL'OPERA.» (120r-120v; anche qui le cc. non sono numerate), divisa in un ordine alfabetico che, ovviamente, subisce naturali modifiche nella traduzione dei nomi in francese. 
veau», 61r), come subire molteplici variazioni, il che ci sembra avere una spiegazione soprattutto quando il traduttore cerca di mantenere la prospettiva offerta dagli aggettivi accompagnanti la parola: «cervellini vani» $(87) \rightarrow$ «cerveaux eventez, vains» (61r); «gli vani cervellini» (87) $\rightarrow$ «Les Cerveaux tels et vains» (61r); «i cervellini curiosi» $(95) \rightarrow$ «les cerveaux curieux» $(71 \mathrm{r})$; «il cervellino» $(87) \rightarrow$ «le cerveau leger» (61r; formula che torna anche altrove, in forma variata: «ces legers de cerveau», 22r, 86r o «cerveaux legers», 86r); «Le donne, secondo il più, hanno i loro cervellini di questa stampa» $(88) \rightarrow$ «Les femmes, pour la plus part, ont leurs esprits frappez à ce coin, et sont legeres de cerveaux» (62r); «simili cervellini» $(97,98) \rightarrow$ «tels ecervelés» $(72 \mathrm{v}, 73 \mathrm{r})$, uso di una forma che Chappuys, si è visto, pareva aver riservato al gruppo dei «cervelluzzi».

$\mathrm{E}$ in relazione ai «Cervelluzzi» che Chappuys introduce le prime varianti nei titoli dei Discours: «Cerveaux moindres» (XVI, 86r) e «Cerveaux de ceste espece susdicte» (XVII, 88v). Quindi la traduzione ritrova equilibrio sulla forma «Cerveaux»: XVIII, 90r; XIX, 91v; XX, 94r; XXI, 95r; XXII, 96v; XXIII, 97v. Si notino le molteplici varianti cui il termine va incontro nel resto della narrazione: «des moindres cerveaux» (86r), «les plus petits cerveaux» (86v, 89r, 98r), «cerveaux» $(89 \mathrm{v}, 90 \mathrm{r}, 91 \mathrm{v}, 94 \mathrm{v}, 96 \mathrm{v}$, 97v, 99r), «entendement» (90v), «esprits» (91v), «esprits et cerveaux menuz» (93v), «menuz cerveaux» $(94 \mathrm{r}, 94 \mathrm{v}, 96 \mathrm{r}$,). Una volta si conserva persino la forma in italiano, «cervelluzzi» (21r), e quando Garzoni rinvia implicitamente ad un particolare tipo di «cervelluzzo», Chappuys esplicita il riferimento, certo per una maggiore esigenza di chiarezza propria della lingua francese: «di questi smemorati» $(118) \rightarrow$ «de ces cerveaux depourveuz» (96r).

Quanto ai titoli dei Discours relativi ai «Cervelletti», Chappuys vi conserva una volta la forma originale «Cervelleti» (21r, scempiamento certo dovuto all'influenza della pronuncia francese) oppure glossa in «les petits cerveaux que nous appellons Cervelleti» (22r); in séguito la traduzione è «petits cerveaux» (XXV, 100v; XXVI, 104v; XXVII, 108v) e «cerveaux» (XXVIII, 112r). Nel testo le forme attestate sono le seguenti: «cerveaux, dicts Cerveletti, petits cerveaux» $(100 \mathrm{v})$, «petits cerveaux» (104r-v, 108v), «cerveaux» (113r, 112r, 113v) e «menuz cerveaux» (114v).

In quattro gruppi si presentano le lezioni dei «Cervelloni» presenti nei titoli: «grands cerveaux» (XXIX, 114v; XXX, 116v; XXXI, 122r; XXXIII, 130v); «grands cerveaux et entendemens» (XXXII, 128v; XXXVI, 154r); «cerveaux et grands entendemens» (XXXVII, 160r); «cerveaux et entendemens» (XXXIIII, 133r). Nel seguito del Discorso si attestano invece: «grands cerveaux» (21r, 114r-v, 115v-116r, 116v, 121v, 130v, 160r-v, 164r-v); «cerveaux» (122r, 128v, 134r); «grands cerveaux et entendemens» (128r, 149v, 154r, 156v); «cerveaux et entendemens» (133r); «cerveaux et grands entendemens» (160r). Per quel che riguarda la poco presente forma «cervellone» $(143,162,163)$, essa è resa con «cerveau» $(128 \mathrm{v}, 163 \mathrm{r})$ e «grand cerveau et entendement» (162r-v).

Le lezioni più numerose, e dalle varie potenzialità semantiche, si riferiscono infine a quelli «chiamati volgarmente cervellazzi, dal consueto parlare di tutta la gente» $(50) \rightarrow$ «appellez vulgairement de nous, Cervellazzi» (21v) e «ceux qui sont du tout sans cervelle, appellez de nous, Cervellazzi» (22v). Nella titolatura dei Discorsi il termine «Cervellazzi» è tradotto: «ceux qui sont du tout sans cervelle» (XXXVII, 164r); «Des ignorans sans cervelle» (XXXVIII, 166v); «depourveuz de cervelle» (XXXIX, 170r ); «despourveuz de cervelle» (XL, 175r; XLI, 180r; XLII, 191r; XLIII, 204v); «despourveuz d'esprit» (LII, 254r); «despourveuz de cervelle, et esprits» (XLIIII, 207v); «despourveuz d'entendement ou ecervellez» (XLV, 212v); «despourveuz de cervelle et entendement» (XLVI, 216r); «despourveuz d'entendement et esprits» (XLVII, 224r); «despourveuz d'entendement et cerveaux» (XLVIII, 231v; XLIX, 234r; LV, 263r); «cerveaux» (L, 242v; LIII, 256r; LIIII, 259r); «despourveuz de cervelle, 
ou Cerveaux» (LI, 251v). Nel Discours la forma più attestata è «cerveaux» $200 \rightarrow$ $213 \mathrm{v} ; 209 \rightarrow 224 \mathrm{r} ; 215 \rightarrow 231 \mathrm{v} ; 217 \rightarrow 234 \mathrm{r} ; 223 \rightarrow 242 \mathrm{v}-243 \mathrm{r} ; 231 \rightarrow 251 \mathrm{v} ; 231 \rightarrow$ 254; $233 \rightarrow 256 r ; 236 \rightarrow 259 r ; 242 \rightarrow 266 v ; 243 \rightarrow 268$ r; numerose le varianti: «ceux qui sont du tout sans cervelle, appellez Cervellazi» (164v), «ceux de ceste maniere» (164v), «ecervellez ou sans cervelle» (164v), «esprits sans cervelle» (166r), «depourveuz de cervelle» (170r); «despourveuz de cervelle» (180r, 191r); «les hommes de cete espece» (175r); «ecervellez» (191r, 205r, 212v, 215v, 234r); «Ecervelez» (204v); «Ecervellez, ou despourveux de cervelle» (205r); «esprits» (207v); «Despourveuz de cervelle ou Ecervellez» (212v); «Esprits despourveuz de iugement» (216r); «destituez d'esprit, et cerveaux» (254r); «despourveuz de sens» (255v), «despourveuz de sens, et cerveaux» (256r; 259r); «esprits et despourveuz d'entendement» (259v); «cerveaux despourveuz de sens et iugement» (263r). L'uso del termine alla forma singolare «cervellazzo» dà «cerveau» $(198 \rightarrow 209 \mathrm{r} ; 227 \rightarrow 248 \mathrm{v} ; 240 \rightarrow 264 \mathrm{v} ; 240 \rightarrow 265 \mathrm{r} ; 241 \rightarrow$ 266r; $242 \rightarrow 266 v)$, con due sole varianti: «un esprit» $(239 \rightarrow 263 \mathrm{v})$ e, per quanto riguarda la forma accompagnata da aggettivo, «il cervellazzo umorista» di Timone $(217) \rightarrow$ «le sauvage humeur» $(233 \mathrm{v})$. Esiste poi il caso in cui Garzoni, pur parlando di un determinato tipo di cervellazzo, usa la forma più generica di cervello. Quando ciò si verifica Chappuys ricorre all'utilizzo della parola alternativa «esprit»: da sola, nel caso di «cervello» (197 e 231) $\rightarrow$ «esprit» (208r e 253r); o in coppia: «cervelli» (198) $\rightarrow$ «Esprits et cerveaux» (209r).

Come si può notare dal contesto di questa classificazione, è interessante anche la dinamica delle varianti aggettivali dei cervelli. Consideriamo il caso dell'aggettivo «vano» cui Garzoni, in periodi serrati, rinvia più volte per qualificare i «cervellini» (87). Chappuys procede ad una resa fedele del qualificante, ogni volta doublé da una variante che ne rafforza e ne puntualizza il significato: «cerveaux eventez, vains» (61r), «Les Cerveaux tels et vains» (61r), «vain et inutil» (61v). Omissione abbiamo invece nel caso di «cervelluzzi di gatta» (50), dove il determinante non è tradotto alla carta 22r ma è altrove reso in «cerveaux de chat» (95r). Il discorso del traduttore si attiene insomma alle potenzialità semantiche dell'italiano, con trasformazioni dovute in parte a coerenza interna, in parte all'esigenza di rendere il testo meglio comprensibile ad un lettore francese.

\section{Mancata 'riscrittura' dei classici}

In tale contesto divulgativo possono essere inserite opportunamente alcune semplificazioni, talora vere e proprie censure, operate a proposito delle tante citazioni latine, o comunque di tradizione classica, e italiane, su cui Garzoni si è detto costruire l'impianto narrativo del Theatro. Il confronto testuale ci dà la certezza che Chappuys riporti fedelmente solo una parte di questo materiale, decidendo altrove di abbandonarlo, con omissioni o riduzioni. Questi tagli, crediamo, hanno come presupposto un alleggerimento del testo e, se escludiamo le censure riguardanti gli autori italiani contemporanei, da supporsi meno noti e quindi forse di minore importanza per il pubblico francese del tempo, le altre non sembrano seguire un filo per così dire equo: lo si vede abbastanza bene da come, in uno stesso passo, un'ampia citazione, presa ad esempio dalle Sacre Scritture, sia riportata per esteso mentre, a distanza di qualche riga, una più breve, ma della medesima fonte, possa venire ridotta o addirittura omessa. Come già aveva avuto occasione di mostrare Marcel Tetel ${ }^{19}$, la traduzione 
delle citazioni latine, fatto non molto frequente all'epoca, costituisce un elemento di novità del testo di Chappuys: si supponeva infatti che un lettore colto fosse in grado di leggere direttamente in latino passi della Bibbia o di autori classici. Sulla base degli esempi che andiamo a proporre si può dunque affermare che la traduzione di Chappuys avesse un intento didattico, per far crescere una comunità di lettori non particolarmente istruita.

Tra i molti esempi fattibili all'interno di questo ampio corpus di riscrittura dei classici, vogliamo solo ricordare (anche per ovvie ragioni di spazio) alcuni nomi che illustrano il carattere enciclopedico dell'opera di Garzoni; il loro persistere nella traduzione di Chappuys, tradotti o meno che ne siano gli esempi, ne conferma la valenza costitutiva e duratura nella cultura dell'epoca. La Bibbia è spesso tradotta, soprattutto i numerosi passi dell'Antico Testamento: Genesi $206 \rightarrow 221 \mathrm{r}$; Esodo $176 \rightarrow 180 \mathrm{v}$; Deuteronomio $145 \rightarrow 131 \mathrm{r}$ (un passaggio relativo alla vendetta, in relazione al quale si traduce anche San Vittore $145 \rightarrow 131 r$ ); Samuele $134 \rightarrow 115$ r; Tobia e Giobbe 177 $\rightarrow$ 182r-v; Salmi $91 \rightarrow$ 66r, $125 \rightarrow 104 \mathrm{r}, 158-159 \rightarrow 157 \mathrm{r}-158 \mathrm{v}, 170 \rightarrow 172 \mathrm{v}, 186 \rightarrow$ $193 \mathrm{v}, 190 \rightarrow 198 \mathrm{v}, 202 \rightarrow 216 \mathrm{r}$; Proverbi $158-159 \rightarrow 157 \mathrm{r}-158 \mathrm{v}$; Sapienza $206 \rightarrow 221 \mathrm{v}$; Sirach $200 \rightarrow 213 v$, $202 \rightarrow 216 v$; Isaia $91 \rightarrow 66 v$, $176 \rightarrow 180 v, 219 \rightarrow 237 v$; Ezechiele $179 \rightarrow 184 \mathrm{v}-185 \mathrm{r}$; Daniele $182 \rightarrow 188 \mathrm{v}$. Ma vediamo anche che, in altri luoghi, Chappuys non fa seguire il testo in latino dalla traduzione in francese: Proverbi $110 \rightarrow 87 \mathrm{r}$, $120 \rightarrow 98 \mathrm{r}, 162 \rightarrow 161 \mathrm{v}$; Ecclesiaste $122 \rightarrow 101 \mathrm{r}, 93 \rightarrow$ 68v; un verso del Sirach $91 \rightarrow$ $66 \mathrm{v}$, mentre tutti i brevi passi precedenti erano stati tradotti, $120 \rightarrow 98 \mathrm{r}, 170 \rightarrow 172 \mathrm{v}$; Daniele $94 \rightarrow 69 v$; Gioele $85 \rightarrow 59$ r. Le stesse note valgono per il meno privilegiato Nuovo Testamento, i cui passi possono essere tradotti: Vangelo secondo Matteo $91 \rightarrow$ $66 \mathrm{v}, 175 \rightarrow 179 \mathrm{v}, 159 \rightarrow 158 \mathrm{v}$; quello secondo Luca $92 \rightarrow 67 \mathrm{r}$; come non tradotti: le parole di Matteo $111 \rightarrow 88 \mathrm{v}$, un passo della seconda Lettera ai Corinzi, $139 \rightarrow 122 \mathrm{v}$. La traduzione delle citazioni latine continua con: San Giovanni 158-159 $\rightarrow$ 157r-158v; Sant'Agostino $178 \rightarrow 184 v$; San Bernardo $177 \rightarrow 182 \mathrm{r}-\mathrm{v}, 190 \rightarrow 198 \mathrm{r}$; un lungo passo di Sant'Ambrogio 182-183 $\rightarrow$ 189v; un aforisma di Galeno $180 \rightarrow 186 v$ come alcune parole di Giovenale $159 \rightarrow 159 \mathrm{r}$. E, per la poesia, con: un verso del poeta e filosofo Lucrezio $139 \rightarrow 122$ r; di Properzio $203 \rightarrow 217$ r; con alcuni versi dell'Eneide di Virgilio $192 \rightarrow$ 201v, $203 \rightarrow 217$ r, $208 \rightarrow 223$ r, $210 \rightarrow$ 226r; con dei rinvii ai sermoni di Orazio $208 \rightarrow 223 v$. Sono tradotti alcuni riferimenti alle epistole di Ovidio $89 \rightarrow 64 \mathrm{r}$, $124 \rightarrow 103 \mathrm{v}, 144 \rightarrow 130 \mathrm{r}, 189 \rightarrow 196 \mathrm{v}, 191 \rightarrow 201 \mathrm{r}, 201 \rightarrow 215 \mathrm{r}, 205 \rightarrow 219 \mathrm{r}, 232 \rightarrow$ $254 \mathrm{v}$; ai versi lascivi di Marziale $107 \rightarrow 83$ r, $136 \rightarrow 118$ r; alle elegie di Tibullo $214 \rightarrow$ 231r; alle satire di Giovenale $187 \rightarrow 194 \mathrm{v}, 201 \rightarrow 215 \mathrm{v}$. E quindi le Historie, traducendo: Sallustio $89 \rightarrow 63 v$ v $185 \rightarrow 192 v$; Seneca $173 \rightarrow 175 v$, $186 \rightarrow 193 v$, $195 \rightarrow 205 v$; Lucano $144 \rightarrow 130 v$; Valerio Massimo $131 \rightarrow 113$ r, $183 \rightarrow$ 190r. E per la retorica, Cicerone $188 \rightarrow 196 v$, $208 \rightarrow 223 v$. Si traduce solo una parte di una citazione da Alessio da Ateneo $(178 \rightarrow 184 \mathrm{r})$ e dalla Consolazione filosofica di Boezio $(184 \rightarrow 192 \mathrm{r})$. Alla traduzione dei testi di questi autori fa spesso séguito, per converso, una loro mancata traduzione. Si pensi alle riduzioni fatte subire ai versi di Virgilio $62 \rightarrow 29 \mathrm{v}, 191 \rightarrow$ $200 \mathrm{v}, 108 \rightarrow 84 \mathrm{v}, 124 \rightarrow 103 \mathrm{r}, 131 \rightarrow 113 \mathrm{r}, 186 \rightarrow 194 \mathrm{r}$ (e quando la citazione è in italiano, Chappuys la riporta in francese $104 \rightarrow 80 \mathrm{r}$ ); a quelli di Ovidio, $74 \rightarrow 44 \mathrm{v}$, $194 \rightarrow 204$ r; nello stesso paragrafo, una volta si traduce un verso di Ovidio (ma che in realtà è di Properzio), per subito dopo non tradurne altri due, pur riportandoli nel testo, $100 \rightarrow 75 \mathrm{v} ; 107 \rightarrow 83 \mathrm{v}-84 \mathrm{r}, 124 \rightarrow 102 \mathrm{v}, 186 \rightarrow 194 \mathrm{r}, 206 \rightarrow 220 \mathrm{v}, 241 \rightarrow 265 \mathrm{v}$; parallelamente, non si traduce Catone $113 \rightarrow 91 \mathrm{r}$, Seneca $110 \rightarrow 87 \mathrm{v}$, Quintiliano 117 $\rightarrow 94 \mathrm{v}$, un verso di Giovenale $192 \rightarrow 201 \mathrm{v}$, un rinvio a Valerio Massimo $208 \rightarrow 223 \mathrm{v}$ e a Claudiano $189 \rightarrow 197 \mathrm{r}$.

Le citazioni dai classici greci avvengono, secondo la moda umanista, in latino $\mathrm{o}$ in italiano. La resa in francese segue i soliti 'criterî': un verso degli inni di Orfeo «Clarior in cunctis Divus splendebat Iason» resta in latino nella versione di Chappuys 
$60 \rightarrow 28$ r; alcuni versi eroici di Omero, che Garzoni citava in italiano, sono invece tradotti in francese, con necessaria modifica della frase italiana introduttiva «scrive quei versi che nella nostra lingua così direbbero» $(88) \rightarrow$ «escrit des vers de ceste substance» $(62 \mathrm{v})$. Quando Omero è citato in latino, lo si riporta nella stessa lingua facendolo accompagnare dalla resa in francese: $174 \rightarrow 178 \mathrm{v}$. Così per le liriche di Saffo, citate in italiano da Garzoni e tradotte in francese da Chappuys $(100 \rightarrow 75 \mathrm{v})$, e per i cantici di Museo «con versi greci che così suonano nell'italiana favella» (88) $\rightarrow$ «en vers Grecs, de ceste substance» (63r), quindi resi in francese. Non tradotti, ma riportati, sono invece due versi di Eraclide Pontico, $103 \rightarrow 78 \mathrm{v}$. I passi di Aristotele (Degli animali, Metafisica, Etica Nicomachea, Politica), che Garzoni cita in latino, non vengono tradotti: $76 \rightarrow 45 \mathrm{v}, 110 \rightarrow 87 \mathrm{r}, 196 \rightarrow 206 \mathrm{v}$, mentre altrove lo sono: $183 \rightarrow$ $190 \mathrm{r}, 198 \rightarrow 210 \mathrm{v}, 210 \rightarrow 225 \mathrm{r}$ (anche se non si tratta del terzo libro dell'Etica, come scrive Garzoni). Si omettono due versi («Fra le stelle del ciel, chiara risplende / la coronna d'Arianna a Bacco moglie») del poeta ellenista Arato $83 \rightarrow 56 \mathrm{v}$, che esprimevano quanto Garzoni aveva appena detto in prosa. E Chappuys traduce Esopo $142 \rightarrow$ 128r, Dionigi l'Areopagita $161 \rightarrow 161$ r, Didimo (citato da Vincenzo di Beauvais) 185 $\rightarrow 193$ r, Epicuro $187 \rightarrow$ 195r. Non traduce invece Plutarco, citato in latino $(115 \rightarrow$ 93r), ma volge direttamente in francese una citazione dal Focione, che Garzoni riportava in italiano $(139 \rightarrow 122 \mathrm{v})$. E a proposito dei «cervelluzzi morti», perché «muti», si rende in francese, senza citarla in latino, la definizione attribuita (ma non riscontrata) a Diogene, «Animali muti» (112) $\rightarrow$ «animaux muets» (89r).

Che Chappuys non dubiti della validità delle citazioni di Garzoni è constatazione abbastanza evidente: la prova è che se l'autore italiano commette un errore nell'identificare una fonte primaria, il traduttore ne conserva la svista. «Maledictus homo qui negligit famam suam» $(145) \rightarrow$ «Maledictus bomo qui negligit famam suam. L'homme est maudict, qui ne fait pas compte de sa renommée» (131v). La frase, in questa forma, non appare nella Sacra Scrittura: crediamo possa ritenersi consonante al precetto «in omnibus operibus tuis praecellens esto I ne dederis maculam in gloriam tuam» (Sirach, 33, 23-24), ma trattasi di congettura. In altro luogo, Garzoni, che cita Geremia, avrebbe invece dovuto riferirsi a Isaia (1, 21); l'errore resta nella traduzione: «Per questo Gieremia profeta parlando di Gierusalemme piena di vizi, disse: 'Quam vilis facta est maeretrix civitas fidelis'» (195) $\rightarrow$ «Pour ceste cause, le Prophete Ieremie, parlant de Hierusalem pleine de vices, a dict, Quam vilis facta es meretrix civitas fidelis: Que tu es devenue vile putain et paillarde, cité fidele» (205v). E quando lo scrittore italiano adatta il testo citato, tali rielaborazioni permangono nella resa in francese. Riportiamo due esempî: il primo da Seneca, «E Seneca disse: 'Muliebre est litigare' (è cosa da donnicciuola vile il contendere e litigare')» (200) $\rightarrow$ «Et Seneque a dit, que Muliebre est litigare, C'est à faire à une vile femme de de [sic] debatre et quereller» (213r), laddove la frase corretta è «Muliebre est furere in ira» (De Clementia, 1, 5, 5); il secondo dalle Etimologie di Isidoro di Siviglia, «Iustitia (come dice Isidoro) est ordo et aequitas, qua homo cum unaquaque re bene ordinatur» $(199) \rightarrow$ «Iustitia (comme dit Isidore,) est ordo et aequitas, qua bomo cum unaquaque re bene ordinatur. / La iustice est ordre et equité, par laquelle l'homme avec chacune chose est bien ordonné et gouverné» $(211 \mathrm{r}-\mathrm{v})$. E la fedeltà, parola per parola, al testo di Garzoni sembra addirittura poter inficiare la competenza linguistica e l'occhio attento del traduttore. Nel Theatro si legge: «Quidam miscelli forte pythagorici I vescuntur in specu altera» (ed. 1585, 80v; l'errore si presenta anche nell'ed. 1583, 80v e nell'ed. 1591, 66v) $\rightarrow$ «Quidam miselli forte Pythagorici, I Vescuntur in specu altera. Certains miserables d'avanture Pythagoriciens, vivent en autre caverne etc.». Chappuys mantiene altera, anziché allera della fonte, ma il senso sembrerebbe risultarne incomprensibile.

Dalla traduzione non sono escluse neppure brevi sentenze di facile compren- 
sione: «Nec musca quidem» $(87) \rightarrow$ «Nec musca quidem, Non mesme la mousche» (62r); «Sola mihi redolet» (104) $\rightarrow$ «Sola mibi redolet, Elle est seule me sentant bon» $(80 \mathrm{v})$; «'Audaces fortuna iuvat' disse il poeta» $(143) \rightarrow$ «Audaces fortuna iuvat: dit le Poete: La fortune aide aux courageux et hardiz» (129v); «in puncto iuris» (197) $\rightarrow$ «In puncto iuris, par le poinct de droict» (208r); «Ob Parni scaphulam» (200) $\rightarrow$ «Ob Parni scaphulam. Pour la petite barque de Parne» (213r); «Multa essent dicenda de fronte illa» (227) $\rightarrow$ «Multa essent dicenda de fronte illa. Il faudroit dire beaucoup de choses d'un tel front» (247v). E Chappuys può anche in questo caso decidere di sopprimere la fonte latina per lasciare solo la versione francese: «per phas et nephas» $(198) \rightarrow$ «par quelque moyen que ce soit» (209v).

A queste testimonianze di traduzione dal latino si uniscono quelle relative alla resa dei testi italiani. Si traduce Boccaccio $(103 \rightarrow 79 \mathrm{r}, 119 \rightarrow 97 \mathrm{v})$, Pietro Bembo $(138 \rightarrow 121 v)$, alcuni versi di Annibal Caro $(64 \rightarrow 31 v-32 r, 68 \rightarrow 37 v)$, di Grotto $(91 \rightarrow 65 \mathrm{v}-66 \mathrm{r})$, del poeta Guidiccione $(92 \rightarrow 67 \mathrm{v})$. Dante, i cui versi sono ogni qual volta riportati per intero o ridotti, spesso non è tradotto: $97 \rightarrow 72 \mathrm{r}-\mathrm{v}, 110 \rightarrow 86 \mathrm{v}$, $125 \rightarrow 104 \mathrm{r}, 188 \rightarrow 196 \mathrm{r}, 192 \rightarrow 201 \mathrm{v}, 214 \rightarrow 231 \mathrm{r}$; lo si traduce invece in due casi: $89 \rightarrow 63 \mathrm{r}$ e $196 \rightarrow 206 \mathrm{v}$. Soffermiamoci in particolare su alcuni esempî di citazione petrarchesca e ariostesca. Petrarca (Canzoniere, Rerum vulgariun fragmenta, Trionfi) è spesso tradotto $(81 \rightarrow 54 \mathrm{r}, 88 \rightarrow 62 \mathrm{v}, 91 \rightarrow 65 \mathrm{v}, 92 \rightarrow 67 \mathrm{v}, 111 \rightarrow 88 \mathrm{r}, 165 \rightarrow 166 \mathrm{r}$, $102 \rightarrow 77 \mathrm{v})$. Ma alcuni suoi versi possono essere anche riscritti senza traduzione: 59 $\rightarrow 26 v$, $99 \rightarrow 75$ r; così ridotti: dei quattro versi di un sonetto (59) si mantiene solo l'inizio del primo verso «Mentre, misera Italia, etc.» (27r); da due versi si passa a uno $(66 \rightarrow 35 \mathrm{v})$, verso che non è tradotto in francese; da quattro a uno, sempre senza tradurlo $(73 \rightarrow 43 \mathrm{r})$; le omissioni comportano naturali adattamenti al nuovo contesto: la cesura di tre versi fa sì che dall'italiano «in quei versi» si passi a «en quelques siens vers» $(74 \rightarrow 44 \mathrm{v})$; così, si eliminano anche frasi «come in quel sonetto ... ed in quell'altro» $(94 \rightarrow 69 \mathrm{v})$. Lo stesso si verifica con l'Orlando furioso dell'Ariosto, che può essere tradotto $(63 \rightarrow 31 \mathrm{r}, 102 \rightarrow 78 \mathrm{r}, 165 \rightarrow 165 \mathrm{v})$, non tradotto pur riportandone i versi ( $92 \rightarrow 67 \mathrm{r}$ ), oppure citato con omissioni all'interno di una stessa stanza $(94 \rightarrow 69 \mathrm{v})$, come modificandone la frase introduttiva, «vagamente spiegolla in quei versi» (95) $\rightarrow$ «l'a proprement declarée et deschiffrée en quelques siens vers» (70r), per in séguito omettere un'ulteriore precisazione, «in quella stanza ... in quella stanza che comincia» $(97 \rightarrow 73 \mathrm{r}$ ); per alcuni versi sulla «lusinghevole Alcina» $(101) \rightarrow \ll l a$ trompeuse et cauteleuse Alcine», Chappuys non riporta i versi italiani, ma ne dà una versione francese presentata in questo modo: «en certains vers de ceste substance et signification» (76r); e sono omessi anche versi di due stanze $(116 \rightarrow 93 \mathrm{v})$, con relativa semplificazione dei rinvii testuali tramite un generico «en plusieurs endroits»; e di «en plusieurs stances» $(233 \rightarrow 255 v)$ Chappuys parla altrove, sempre in caso di omissioni. Analoghe riduzioni si segnalano in altri luoghi:145 $\rightarrow 131 v, 211 \rightarrow 227$ e $239 \rightarrow 264 \mathrm{r}$.

L'esigenza di divulgazione e la portata didattica del testo francese spiegano anche, ci sembra, le riduzioni operate dal traduttore sugli scritti di autori moderni e contemporanei. Dell'Anguillara sono spesso riportati i versi in forma ridotta, ma comunque non tradotti: $106 \rightarrow 83$ r, 232-233 $\rightarrow 255 \mathrm{v}, 121 \rightarrow 100 \mathrm{r}, 203 \rightarrow 217 \mathrm{v} ; 240 \rightarrow$ $264 v$. Sono omessi quattro versi di Goselini $(85 \rightarrow 59 \mathrm{r})$, con conseguente modifica della frase «in quel sonetto che comincia» $\rightarrow$ «en un sien sonnet»; si riducono, senza tradurli, i versi di Molza $(209 \rightarrow 224$ r, $215 \rightarrow 231$ r), con passaggio da «in quei versi» $(85) \rightarrow$ «en vers qui commencent» (59v); sono omessi delle terzine di Varchi $(86,87$ $\rightarrow 60 \mathrm{v})$, due versi del «gentil Remigio Fiorentino» $(94 \rightarrow 69 \mathrm{v})$, mentre non riportati risultano alcuni versi di Giulio Camillo e Pietro Gradenico, con conseguente fusione delle frasi con cui Garzoni li presentava: «Così il dottissimo Giulio Camillo ve la pose nel sonetto che comincia ... Ed il clarissimo Pietro Gradenico in quello che 
principia» $(100) \rightarrow$ «Iules Camille, et Pierre Gradinique l'ont mis aussi en leurs vers» (75v-76r). Chappuys elimina inoltre alcuni versi di Fabio Galeota e Giulio Morigi $(215 \rightarrow 231 r)$, ne omette tre di Giovan Battista Rossi $(160 \rightarrow 160 r)$ e riporta, ma non traduce, tre versi di Agostino Augurello sulla pietra filosofale $(218 \rightarrow 235 \mathrm{v})$, così come non traduce, bensì riduce notevolmente, un madrigale di Giacopo Bonfadio (166 $\rightarrow 166 r$ ), come pure dei versi di Angelo Poliziano $(234 \rightarrow 257 \mathrm{r}$ ).

Stesso intento divulgativo riteniamo abbiano anche le semplificazioni relative ai nomi di personaggi illustri. Nel testo di Garzoni, Marco Tullio Cicerone può essere indicato nelle sue varianti come M. Tullio $(60,71,78,86,137,188)$, Tullio (81, 84, $126,128)$ o Marco Tullio $(117,184)$; Chappuys vi rinvia ogni volta col nome di «Ciceron» $(28 \mathrm{r}, 49 \mathrm{r}, 53 \mathrm{v}, 57 \mathrm{v}, 60 \mathrm{v}, 95 \mathrm{r}, 105 \mathrm{r}, 109 \mathrm{v}, 119 \mathrm{v}, 174 \mathrm{r}, 191 \mathrm{v}, 196 \mathrm{v}, 203 \mathrm{r}, 207 \mathrm{r}, 208 \mathrm{v}$, $223 v, 225 r$. Analoga soluzione volgarizzante per l'altrettanto noto Publio Virgilio Marone: «l'Eneida di Marone» $(147) \rightarrow$ «l'Eneide de Virgile» (134v). E quando il cognome è accompagnato da un connotativo, Chappuys tende ancora a semplificare: «Teocrito chio» $(124) \rightarrow$ «Theocrite» $(103 \mathrm{r})$; «Seneca il morale» $(186,198) \rightarrow$ «le Philosophe Seneque» (193v, 210r); «Ovidio poeta» $(57,205) \rightarrow$ «Ovide» (24v, 220v); «il commendatore Annibal Caro» (64) $\rightarrow$ «Annibal Caro» (31v); «Valentiniano Cesare» $(166) \rightarrow$ «'Empereur Valentinian» $(166 \mathrm{v})$.

\section{Norme linguistiche}

Un tratto che marca vistosamente il linguaggio usato da Garzoni nel Theatro è il ricorso ad un ampio registro di termini, intensificato certo dalla volontà di connotare l'espressione, spesso ipertrofica, della voce dei pazzi. Mentre Garzoni tende ad un linguaggio elaborato, Chappuys preferisce una costruzione che semplifica la portata metaforica del testo italiano, in ottemperanza ai fini didattici del testo quanto ad una esigenza, senz'altro individuale, di chiarezza descrittiva ${ }^{20}$. Si vedano, tra i molti esempî: «più ch'aquile alteri spiegano il volo» $(47) \rightarrow$ «volent plus haut que les Aigles» (17r); «col lume deputato della luna» (57) $\rightarrow$ «par la lumiere aussi de la Lune» (25r); quando il poema di Omero è definito «parto» (76), Chappuys parla di «conception et fruict» (46r); «più tosto sotto indegno silenzio trapasso che imbrattar le lodi di quei con queste labbra rozze, infaconde ed inette» (79) $\rightarrow$ «ie passe plustost sous un indigne silence, que de souiller ou obscurcir les louanges d'iceux, par ce que ma plume rude, non faconde et inepte en pourroit escrire» (52r); «Perciò nacquero a' virtuosi e nobili appresso a' Romani tante sorti di corone» $(83) \rightarrow$ «Et pour ceste raison les Romains donnoyent aux vertueux et nobles, tant de sortes de coronnes» (56r); scrivendo «De' cervelletti ciarlieri, linguacciuti e mordaci» (Discorso XXV) e dei danni causati dalla lingua, «del suo mordere» (124) $\rightarrow$ «de son mesdire» (103r) e «questa sfrenata mordacità medesima» (124) $\rightarrow$ «ceste mesme licence effrenée de mal parler» (103r); «gli ordini vanno a spasso» $(141) \rightarrow$ «l'ordre bany» $(125 \mathrm{v})$. E il traduttore tende anche ad esplicitare la sineddoche: «un bellissimo legno» (76) $\rightarrow$ «un tres-beau vaisseau» $(46 v)$, e «gli ficcò dentro al cervello» $(111) \rightarrow$ «luy ficha en la teste» (88v).

A circonlocuzioni e perifrasi Chappuys ricorre per evitare parole o espressioni troppo crude; limitandoci ad alcuni esempi significativi: «per troppo stomaco della

(20) Vedi V. Pompejano Natoli, La follia 'ospitalizzata'. Dal trattato di Tommaso Garzoni al teatro di Charles Beys, in «Studi di letteratura francese», XIX, op. cit., p. 240 e sg. Sul paziente «travail de rabotage» effettuato da Chappuys nelle sue traduzioni, con particolare attenzione per la figura dell'iperbato, vedi M. A. LORGNET, La figure de l'byperbate comme expression du sublime: à propos des traductions du Roland Furieux au XVI siecle, in Miscellanea in onore di Liano Petroni. Studi e ricerche sulle letterature di lingua francese, Bologna Clueb, 1996, pp. 17-29. 
natura loro ... non con minor stomaco» (96) $\rightarrow$ «pour l'horreur de leur nature ... avec non moindre borreur et fascherie» $(71 \mathrm{r}, \mathrm{v})$; «puzzano d'una grandezza stomacosa da ogni parte» (97) $\rightarrow$ «toute chose leur vient à contrecœur, et ne trouvent rien à leur gré» (72v); «un grammatico che è un ciancione» (140) $\rightarrow$ «un Grammerien qui ne fait que iaser et ne dit rien qui vaille» $(124 \mathrm{v})$; «ne' tripudi lascivi» (177) $\rightarrow$ «aux danses» (183); «a schifo» (178) $\rightarrow$ «en grande horreur» $(183 \mathrm{v})$; «dissoluzioni della gola. Ob gola ...» (180) $\rightarrow$ «dissolutions de la bouche. O gourmandise» (186r); «la verginella» $(183) \rightarrow$ «la gentil femme» $(183 \mathrm{r})$; «il misero cadavero» di Polidoro (189) $\rightarrow$ «le miserable corps» (197v); «Benché non è meno bestiale quella proposta» (217) $\rightarrow$ «Bien que ne soit pas moins brutalle la proposition» $(233 \mathrm{v})$ (l'aggettivo «bestiale» torna ad essere tradotto con «brutal» anche nel Discorso LII, 254r, a proposito dei «Cervellazzi pazzi, furibondi e bestiali»). Lo stesso avviene per la resa dei proverbi o modi di dire propri dell'italiano che, nel passaggio al francese, subiscono necessariamente una modifica, affinché siano accessibili al nuovo pubblico: «a' grilli della terra, come si dice per commun proverbio» $(90) \rightarrow$ «aux fantasies et fanfreluches de la terre» $(64 \mathrm{v})$ e «non bisogna saltar (come si dice) di scala in tetto e di palo in frasca». (92) $\rightarrow$ «il ne faut pas estre leger et inconstant de pensée et d'actions» (67r). Si noti inoltre l'inevitabilità di una perdita nella traduzione: nel capitolo «De' cervellazzi d'astrologo», Garzoni scrive «Ma ben moltissimi sono non astrologi, ma stralocchi» (229), dove stralòcchio (voce di area settentrionale composta dal lat. extra e da occhio con inserzione dell'articolo) è alla base di un jeu de mots con (a)strologo $o^{21}$; il gioco linguistico astrologo/stralòcchio viene meno in francese: «Mais bien plusieurs, et en grand nombre, se trouvent non Astrologues, mais ignorans» (251v).

Tra i diversi tipi di cambiamenti linguistici grande spazio è occupato dai processi di esplicitazione (coppie affini o sinonimiche, glosse con vocaboli o sintagmi più familiari) e di semplificazione semantica (riduzioni che comportano talvolta la perdita o la riduzione della figura), scelte che dipendono dalla volontà di rendere più comprensibile il testo nella lingua di arrivo ma anche dal più misurato procedere del traduttore; basti qui qualche esempio fra i numerosissimi: «pare a costoro» $(47) \rightarrow$ «il semble telle maniere de gens» (17v); «di costoro» (47) $\rightarrow$ «de ces hommes là» (17v); «il vento Austro» (118) $\rightarrow$ «le vent Auster ou Meridional» (96v); «giorno e notte e per freddo e per caldo e per pioggia e per venti» $(121) \rightarrow$ «iour et nuict, hiver et esté, et par la pluie et par les vents» (99v); «chi si tiene d'essere 'muylindo', come dice lo spagnuolo» (129) $\rightarrow$ «qui s'estime estre fort gentil, et Muy lindo, comme dit l'Hespagnol» (111r); «l'epulone» (180) $\rightarrow$ «le banqueteur et gourmand» (186v); «le pasquinate» (204) $\rightarrow$ «les Pasquinates, ou libelles de difame» (218v); la virtù è «dogma di tutti i savi» (236) $\rightarrow$ «enseignement de tous les sages» $(259 \mathrm{v})$; «Nella modestia della mente» $(85) \rightarrow$ «Et mesmes la vertu de l’homme consiste en la modestie de l'esprit» (59r); «disse un giudicioso spirito» (88) $\rightarrow$ «selon le dire d'un homme d'esprit et iugement» (62r); «le vane» $(89) \rightarrow$ «les femmes vaines» (63r); «non li piegava le ginocchia come gli altri» (96) $\rightarrow$ «il ne luy plioit les genoux, et ne luy faisoit reverence comme les autres» (72r); «di non picciola leggerezza» (98) $\rightarrow$ «d'une grande legereté» (74r); «Io non dirò quante lagrime gettano gli infelici, ché le lagrime di Didone per Enea» $(104) \rightarrow$ «Ie ne diray combien de larmes iettent les pauvres et infortunez amans: car les larmes de Didon, pour l'amour d'Aenée» (80v); «Dante per Beatrice, il Petrarca per Laura» $(105) \rightarrow$ «Dante, pour l'amour de Beatrix: et Petrarque, pour l'amour de Laure» (81r); «Non fu minor quella» (113) $\rightarrow$ «La sottise ne fut pas moindre» $(90 \mathrm{v})$; «a' suoi incipienti» $(123) \rightarrow$ «à leurs disciples commenceans à recevoir

(21) Vedi Grande Dizionario della lingua italia$n a$, fondato da S. BAtTAgLia, diretto da G. BÀrberi 
doctrine et instruction» (102r); Apollonio Tianeo «entrò ne' Persi, passò il monte Caucaso, scorse gli Albani, gli Sciti, i Massageti, penetrò gli Indi» (143) $\rightarrow$ «entra au pais des Perses, passa le mont Caucase, courut le pais et regions des Albains, des Scithes, et Massagetes, traversa les Indes» (129r); l'imperatore Adriano fu «peritissimo dell'aritmetica e della geometria, dipinse egreggiamente» $(147) \rightarrow$ «tres-excellent et entendu en l'Arithmetique et Geometrie. Il fut brave peintre» (133v); «e fanno uno strepito, un romore, come se tu facessi un latin falso» (200) $\rightarrow$ «et font un bruit et tumulte aussi grand, que si tu avois commis un solecisme et incongruité, à parler Latin» (213v); «la calamita» (224) $\rightarrow$ «la Calamite, ou pierre d'Aimant» (243v); «e a guisa di cingari» (227) $\rightarrow$ «et en maniere de Bohemiens, comme on les appelle communement, qui courent par pais» (248r); «Tutti non sono gli Atlanti che possino sostenere l'Olimpo con le spalle» (229) $\rightarrow$ «Tous ne ressemblent à Athlas, pour pouvoir soustenir et supporter le ciel de leurs espaules» (251r-v); «un paio di can corsi» (130) $\rightarrow$ «une couple de chiens» (111v); «città d'Esperia» (143) $\rightarrow$ «villes d'icelle» (128v); «da pecore lombarde e da castroni di Puglia» (201) $\rightarrow$ «en bestes brutes» (214r). Probabilmente col desiderio di eliminare la ripetizione propria dell'italiano si spiegano i cambiamenti legati al termine «ghiribizzo»: «secondo il proprio ghiribizzo» (237) $\rightarrow$ «selon sa propre volonté» $(260 \mathrm{v})$; «oh ghiribizzi erronei» (237) $\rightarrow$ «ô fantasies estranges et pleines d'erreur» (261r); «novi ghiribizzi» (238) $\rightarrow$ «nouvelles fantasies» (262v).

$\mathrm{Da}$ Garzoni a Chappuys il testo conserva la sua interezza. L'ampio ricorso ai cataloghi, proprio dello scrittore italiano, viene rispettato dal traduttore, il quale cerca di rendere il più fedelmente possibile ogni termine delle lunghe e numerose liste. Poche le eccezioni, che qui vogliamo riportare. Nell'elencazione delle varie scienze (Discorso XXXIV), Chappuys riduce soltanto lo spazio dedicato alla geometria: «prencipe e madre di tutte le discipline, sapranno divisar de' punti, delle linee, delle superficie, de' corpi, delle forme, de' spazi, delle misure» (148) $\rightarrow$ «mere de toutes les sciences et disciplines, ils te sçauront deviser des poincts» (136r). Nel caso di voci tecniche o specialistiche, il traduttore scioglie il termine italiano [«de' venti (levante, ponente, ostro, tramontana, greco, sirocco, garbino e maestro)» (152) $\rightarrow$ «des Vents, du Levant, Ponent, Midy, Septentrion, du Grec, Meridional, Garbin, venant d'Afrique et orageux, et du vent qui souffle du costé du Soleil couchant, dict Corus» (142v)] o semplifica con omissione [per la cucina, «il persciuto di Chio» $(153 \rightarrow 149$ r); scrivendo delle donne, che Garzoni inserisce nel capitolo «De' cervellini vani», pur traducendo con fedeltà la lista delle principali attività loro attribuite e i nomi degli oggetti solitamente utilizzati nel quotidiano, Chappuys omette soltanto «ampolle, bossoli» (88) $\rightarrow$ «des phioles» (62r)]. Larghe semplificazioni sono date per la preparazione degli elisir alchemici. Se è vero che la curiosità «De' cervellazzi alchimistici» si esperimenta in ambiti articolati ${ }^{22}$, tra le particolari sostanze usate nelle ricette degli alchimisti Chappuys non traduce solo «risalgaio» $(219 \rightarrow 236 \mathrm{r})$, il sublimato corrosivo che nel Mondo savio e pazzo del Doni il magistrato somministra a chi non ha voglia di lavorare, tra i minerali «vitriolo, marchesita, salnitro, verderame» (219) $\rightarrow$ «Vitriol, Salnitre et autres» (236r-v), tra i succhi d'erbe esclude la «saponaria» e, tra le medicine, il «succo d'oppio» (236v). E quando Garzoni scrive che «si pone in crosolo ogni cosa in foggia di pasta lutata (col loto pazzia, ch'io non dirò sapienza) coperta con tegola, senza rispiraglio di sorte alcuna» (219), Chappuys semplifica, «se met tout cela en un vaisseau, en forme de paste, bien bousché, sans aucun vent» (237r), omettendo anche il rinvio alla pazzia intesa come sapienza, cui ci siamo precedentemente riferiti. E al ri-

(22) Sulla quantificazione degli elementi alchemici e la moltiplicazione dei segni necessaria alla sua mise en écriture, entrambe prove di «énuméra- tion chaotique» e indizio di «écriture en folie», vedi C. Ossola, art. cit., p. 191. 
guardo «De' cervellazzi d'astrologo», in una lunga lista di sapienti Chappuys esclude soltanto il termine «Alfragani» $(228 \rightarrow 249$ r), mentre ricorre all'uso di un' espressione più generica quando Garzoni definisce l'ignorante «un'oca d'intelletto ... un cucco di giudicio, un alocco di senno e di sapere» (167-168) $\rightarrow$ «un oiseau, d'entendement, ... un cocu, de raison et discours, et un chat-huant ou un Hibou de sens et sçavoir» $(168 v)$. Le semplificazioni si rivelano necessarie anche in ambito militare:

«quelli che stanno su 'l quistionare, ch'ogn'ora vanno imaginandosi con che arte, con che inganno, con che stratagema il nimico si possi porre a dormire, se i balestrini veronesi siano atti, se le scattole modonesi faran l'effetto» (224) $\rightarrow$ «ceux qui sont sur les questions, lesquels à toute heure vont imaginant, par quel art, par quelle ruse, et par quel stratageme, l'on puisse accueillir et surprendre l'ennemy dormant» (243v) [le altre due espressioni, riguardanti termini di arte tecnico-militare, non sembrano registrate nei dizionari];

«le berette sopra gli occhi, con le penne alla guelfa o alla gibellina; i fiori nell'orecchia, o alla destra o alla sinistra; $i$ zucchetti o le secrete di ferro in testa, li piastrini o giacchi del continuo indosso; le manopole o $i$ guanti da presa in mano; le spade, o gli verdughi da lato; le scimitarre o i pistolesi sotto gli arcobusetti proibiti, o i balestrini nelle brache, e, insomma il diavolo nella testa e nel cervello» (235) $\rightarrow$ «leurs chapeaux sur les yeux, avec la plume au vent, et les rebras retroussez, le bouquet à l'oreille, ou droicte, ou senestre: le secret cabasset en teste: la maille sur le dos: les espées et dagues au costé, les pistoles, dessouz la cappe et autres bastons deffenduz: et en somme le diable en la teste, et au cerveau» (258v).

Le perdite sono compensate da nuove figure, quasi sempre coppie sinonimiche, secondo quel fenomeno di duplicazione riconosciuto anche da Marcel Tetel, il quale muove da un esempio di «réduplication» per avanzare l'ipotesi di una pretesa a «un didactisme plus poussé» del testo di Chappuys ${ }^{23}$. Il fenomeno della (doppia) duplicazione - che può riguardare il sostantivo, l'aggettivo o il verbo -, costituisce una costante molto frequente della resa in francese del Theatro. Tra i numerosi esempi, ci pare utile ricordare almeno alcune forme di doppia duplicazione che confermano l'interpretazione del testo data da Tetel: «da questa alacrità non mediocremente refocillati» $(65) \rightarrow$ «fort recreez et restaurez, par cette gayeté et allegresse» (33v); «un brutto forestiere» (67) $\rightarrow$ «un bideux et diforme estranger et hoste» (36v); «giuochi proibiti ... tutti i tripudi» $(176) \rightarrow$ «ieux probibez et deffenduz ... toutes danses et trepignements» (181r); «le dili$z i e$, ed altri gli stenti» (199) $\rightarrow$ «les plaisirs et delices, veu que les autres ont les tourmens et peines» $(211 \mathrm{v})$; «perché la libertà di scorrere a tuo modo, stando gli altri legati alla catena della servitù? perché tu trionfi del tuo appetito privato, patendo gli altri anco nelle cose necessarie, come sovente fanno? perché portare in mano quella bacchetta, agli altri sì severa, a te stesso sì parca e sì misericordiosa?» $(199) \rightarrow$ «pourquoy veux tu la liberté de courir ça et là à ta fantasie, estans les autres liez et attachez à la cadene de la servitude et subiection? pourquoy demandes tu ta particuliere volonté et appetit, veu que les autres endurent et patissent mesmes és choses necessaires, comme ils font souvent? Pourquoy veux tu porter ceste verge et sceptre en la main, aux autres tant severe, et à toy mesme tant doux et misericordieux?» $(211 \mathrm{v}-212 \mathrm{r})$; «tu onori le giovane ... tu sublimi gli vecchi» $(86) \rightarrow$ «tu pares et embellis le sexe feminin ... tu bausses et esleues les vieillards» (60r); «per ingannar le sciocche donne» (170) $\rightarrow$ «pour decevoir et abuser les sottes et simples femmes» (171v).

Si osservi inoltre un caso di tripla duplicazione riguardante alcune caratteristiche proprie «De' cervelli faceti», posti nel «quarto luogo del teatro», i quali «c'hanno

(23) Vedi art. cit., p. 224. Sulla iteratio sinonimica vedi anche P. DE CAPITANI, Un traducteur français..., 
una certa piacevolezza inserta in loro, facilissima ad acquistar la grazia altrui» (67) $\rightarrow$ «ont en eux une certaine gayeté et gaillardise, tres-facile et propre pour acquerir la faveur et grace d'autruy» (36r). Questo ultimo esempio permette di ricordare che il Discorso IV offre un interessante caso di rapporto tra lingua e cultura. Vi compaiono infatti parole che contribuiscono a inserire il Theatro nella cultura del tempo: urbanità, dilettazione, piacevolezza, grazia, concetti che rendono i cervelli piacevoli e faceti, appunto. Nel testo francese, il lessico si precisa in grace et gayeté, delectation, gayeté et gaillardise, faveur et grace, per creare dei cerveaux plaisans et gaillards (all'aggettivo gaillards Chappuys ricorre forse per ovviare la ripetizione del frequente facetieux). E la stessa funzione di richiamo agli indirizzi culturali dell'epoca è data dalla scelta degli autori menzionati: dal modello Aristotele, con la virtù eutrapelia di cui si legge nell'Etica, a Stefano Guazzo e La civil conversazione, da Pietro Bembo alla 'disinvoltura' del Cortegiano del Castiglione. Scopriamo insomma parole-testimonio e protagonisti-modello che ci permettono di verificare, una volta di più, un'affinità di gusto e di pensiero nella cultura dominante in Italia e in Francia alla fine del Cinquecento.

\section{4. «Unicuique suum»}

Con queste note, crediamo di aver sottolineato l'importanza della traduzione del Theatro de' vari, e diversi cervelli mondani per gli studi sulla traduzione e adattamento cinquecenteschi. Gabriel Chappuys, seguendo da vicino Tomaso Garzoni, presenta sotto gli occhi dei francesi le varie specie di «cervelli nominati al mondo», con la differenza che, se il Canonico mantiene il discorso sempre ad un alto livello di erudizione, il traduttore sembra invece perseguire lo scopo di avvicinare un pubblico decisamente meno colto. Non che il testo di arrivo faccia venir meno i possenti repertori utilizzati nell'originale, né sono trascurate le abbondanti citazioni volte a rinsaldare gli argomenti toccati di volta in volta: ma i tagli effettuati agli esempî nella loro interezza, la ripetuta traduzione delle numerose citazioni, spesso omettendo la lingua originale, nonché una resa stilisticamente appianante, come è indispensabile per entrare a contatto con un maggior numero di lettori, segnano un dato nuovo tra i problemi della volgarizzazione. La modestia didattica di Chappuys, sostenuta dall'apprensione di far conoscere il libro italiano in terra francese, rende stavolta l'essenza del Theatro inaspettatamente incompleta. Questo è il punto paradossale di una traduzione pur così in apparenza accurata e paziente.

Nel Theatro Garzoni ha saputo ben classificare e posizionare i varî 'temperamenti'. Fedele alle regole del suo tempo, lo scrittore è portato a cogliere i materiali concreti più utili al lavoro intrapreso che, per riprendere la definizione garzoniana dell'Iliade, possiamo così definire come «un tascone pieno di denari» (77). Senza dubbio consapevole del valore di questa «bourse pleine d'argent» (48v), Chappuys vi attinge, in maniera informata e attenta. Tuttavia, lo si è visto, la distribuzione di quanto maneggiato non avviene secondo criteri univoci, lungi dunque dal far ottenere l'effetto sperato. Forse Chappuys avrebbe voluto conservare l'intero corpus testuale anche nella sua resa francese ma, se un responsabile deve essere cercato, si potrebbe pensare all'intervento dell'editore (la probabile richiesta di maggiore brevità nasconde, nemmeno troppo velatamente, il risparmio di natura economica), che gli avrebbe impedito il progetto di una più giusta fedeltà all'originale. Ci sarebbe stato bisogno di «una costante e perpetua volontà di dare a ciascuno il suo» (Discorso XLIIII), ma sappiamo bene, come ci ricorda Anna Bettoni, che già all'epoca le motivazioni editoriali dettano non solo l'immissione dei testi sul mercato ma hanno anche una parte importante nell'organizzazione del lavoro stesso: le differenze non sono molto diverse da oggi. 